\title{
THE DEVELOPMENT OF METEOROLOGY AS AN EXACT SCIENCE*
}

\author{
By Dr. O. G. SUTTON, C.B.E., F.R.S.
}

$I^{\mathrm{T}}$ would be idle to pretend that meteorology, although it uses the language of physics and mathematics, has yet acquired the status of an exact science in all its branches. To take an obvious example, routine weather forecasting from the synoptic chart as yet involves very little actual calculation, and the reasoning with which the forecaster judges future developments is qualitative rather than quantitative, although it is essentially rooted in physics. My aim here is to attempt a critical examination of the part played by mathematics in meteorology - a stocktaking, to see how far the adjective 'exact' applies to meteorological science in the year 1954 .

Mathematics has two main uses in applied science. The first is to provide an objective method of summarizing experience - to act as a 'memory'. This is what is done when an empirical formula is devised to represent, say, the rate of evaporation from a lake, or when a Fourier series is fitted to the diurnal variation of temperature. It is a humdrum use of mathematics, but essential in a subject such as meteorology, in which, at times, the sheer mass of observational data threatens to obscure the physical processes at work.

The second main use of mathematics in physical science is to make predictions. However, it is impossible to solve real problems of physics by mathematics ; we can solve only certain ideal problems which have affinities with real problems. In many problems of physics the agreement between the numbers which appear in the mathematical solution and the pointerreadings of the laboratory instruments is so close that the illusion of having solved a real problem is almost overwhelming; but even in the simplest situations the agreement with observation cannot be perfect. In meteorology, the gap between the real situation and the ideal problem is much greater than in laboratory physics, so that it may appear sometimes that the mathematical meteorologist scarcely lives in the same world as his 'practical' colleague; but the difference is one of degree only, and not of principle.

A science may be regarded as 'exact' when it is possible to make observations of high precision on the state of a system, and when the solutions of certain mathematical problems correspond closely to the numbers indicated by physical measurements. How close the correspondence needs to be is largely a matter of judgment, based on the reliability and objectivity of the observations. Obviously, the criteria which apply to, say, spectroscopy are much too severe for meteorology, and we may be well satisfied with a meteorological 'theory' in which the difference between the observed and calculated values is not more than, say, 10 per cent. There are very few meteorological theories which satisfy this criterion at the present time.

\section{Nature of the Meteorological Problem}

Meteorology is concerned with physical processes taking place in the atmosphere, a shallow ocean of gases held to the surface of the earth by the force of

- Substance of the presidential address delivered before the Royal Meteorological Society on April 28 . gravity. The first picture of the atmosphere is that of a shallow gaseous fluid heated from below, which immediately suggests certain problems in natural convection that, to date, have yet to be solved satisfactorily even for the relatively simple conditions which can be realized in the laboratory. (In addition, there is the very significant complication that the convective system is based on the spinning earth.) For the meteorologist, the most important constituent of the atmosphere is water vapour, which supplies an effective means of taking up and releasing heat in enormous quantities. The result is to complicate tremendously the convective system formed by the sun, the atmosphere and the earth's surface. The system, which can scarcely be isolated from the universe as a whole, is one in which the state of the lower boundary influences, and is influenced by, the condition of the gaseous fluid resting upon it. The meteorologist thus has to deal with the vagaries of an open system which is strongly cross-linked. Almost every element of the system reacts significantly with every other, and non-linearity, the bugbear of the mathematician, is the rule rather than the exception. There is a strong temptation to propound soluble problems in physics which have some relation to atmospheric processes and to call the result 'dynamical meteorology'-a type of academic exercise which is only too familiar in the older books on the subject. The real advances are not made in this way, and the essential step lies in discovering the framework of the real problem. It is unprofitable to formulate first an artificial but soluble problem and then to look for occasions when the atmosphere appears to conform.

We may distinguish two classes of problems in meteorology. In the first class are problems, like evaporation, which have nearly everything in common with laboratory problems except the possibility of complete control. In the second class are problems which have little or nothing in common with labor. atory experience, such as those which appertain to the development and movements of the large-scale pressure systems of the middle latitudes. Mathematics can be applied with profit in both classes, but with very different criteria of success.

\section{Evaporation}

Evaporation is a typical problem of the first type, which can be approached in two different but legitimate and profitable ways: (i) by an examination of the mechanism of the process, disregarding the economics, and (ii) by evaluating the onergy balance, disregarding the mechanics.

Evaporation from a semi-infinite area poses a typical boundary-value problem, of a type familiar in the theory of conduction of heat. The earliest treatment is that of Jeffreys, in which the total evaporation from an area extending a known finite distance downwind is given by an explicit expression involving the wind speed, dimensions of the area and the eddy conductivity $K$. If $K$ were a true universal constant, knowledge of the wind speed, the relative humidity, temperature of the surface and the size of the area would enable the meteorologist to calculate 
the amount of water carried over the downwind edge of the area in a given time; but this solution cannot be used to make predictions, because of the uncertainty regarding the value of $K$. In 1934 I published an extended treatment of the semi-infinite area problem, which took into account the variability of wind speed with height and expressed the effect of turbulence, not by a fictitious constant, but in terms of measurable quantities. This solution could be used to make predictions, and soon afterwards Dr. F. Pasquill showed that the measured total of evaporation from small saturated surfaces agreed with the mathematical forecast to within about 10 per cent.

At this stage in the investigation this work was not peculiarly meteorological, for the mathematical treatment and the subsequent verification were all on lines familiar in any physical problem. Since then, there has been one notable attempt, in America, to extend the investigation to the meteorological scale, at Lake Hefner, in which it is concluded that two of the theoretical formulæ tested "gave good results" in predicting the rate of evaporation from measurements of wind, temperature and humidity.

The study of evaporation is instructive because it illustrates very clearly the way in which laboratory physics of the orthodox type merges into meteorology, but with a substantial loss of precision as it does so. This particular problem, approached in the classical manner, is yielding to research in a fashion well known to scientists since the seventeenth century, and there can be little doubt that as we discover more about the mechanics of turbulence we shall get better agreement. All observations of physical quantities contain random fluctuations covered by the generic term 'noise', which limit the precision with which the result can be stated. The mathematical problem effectively relates to an ideal, noise-free system. In meteorology, the 'noise'-level is always much higher than in laboratory physics, and meteorologists must accept as satisfactory agreement between quantities which are, to the laboratory physicist, rather crude averages.

The alternative way of attacking this problem would scarcely recommend itself to a laboratory physicist, but it has considerable merits in meteorology. The 'energy-balance' method consists in first evaluating or estimating all the terms in the transformation of energy except one, that which represents the heat used up in evaporation, which is finally determined as a difference. This method also has given good results.

Evaporation is typical of a large class of meteorological problems in which one may look forward with confidence to ultimate success. In such studies the state of the atmosphere is prescribed, and it is asked how this affects certain physical processes. Provided that a sensible view is taken of the desirable accuracy, there seems to be no reason why such questions may not be finally settled. It is otherwise with problems arising in the phenomenon of weather.

\section{Mathematics in the Synoptic Problem}

The central problem of meteorology is that of forecasting weather. Attempts to bring this within the discipline of mathematics have been, in the main, by the study of 'model' atmospheric disturbances, by statistics, and by the equations of hydrodynamics. Analyses of synoptic situations based on analogies with motions studied in the classical hydrodynamics flourished in the early years of this century. Such 'mechanical' models are now of historical interest only. They represent an excessive idealization, for essentially they are two-dimensional models given a three-dimensional appearance.

The three-dimensional character of the middlelatitude disturbance begins to emerge, however, in the Norwegian concept of the evolution of the depression as a development of wave-like perturbations of the polar front. We know now that the true Bjerknes depression is somewhat rare, and that the sequence of events described in the earlier papers represents a very considerable idealization of what actually occurs; but even so, there is every reason to be grateful to the Bergen school for showing, by precept and practice, the value of air-mass analysis in routine forecasting.

The statistical approach has attracted, possibly, more workers than any other; but to-day it is doubtful if anyone (apart from a few enthusiasts) would claim that the results obtained are commensurate with the effort expended or that the method merits further serious study. The search for empirical periodicities and correlations is not only unsound but also dangerous, in that it tends to create a state of mind approaching that of the 'true believer', who sees examples to support his creed in every case he examines. As regards the possibility of using harmonic analysis in forecasting generally, the conclusion seems to be that the application of statistical theory to pressure-time series for the purpose of forecasting weather is a misuse of a mathematical tool. The prime purposes of statistical theory are to examine data for internal consistency and to weigh the evidence for a previously formulated hypothesis; and in meteorology, as in any other science, it is essential that the research have a sound physical basis before any statistical approach is attempted.

The 'mechanical model' and 'statistical' methods represent two widely different approaches. There is, however, a middle way which seems to hold out considerable hope, namely, the direct attack on the problem by means of the equations of hydrodynamics. The limitations of the method are very serious. It is not so much the complexity of the situation which appals the mathematician, but the fact that the problem may be indeterminate. It seems that at times the initial conditions may be insufficient to determine a unique solution. The atmosphere is so nearly unstable that disturbances which are below the threshold of observation at one time, or which are so small initially that they are removed in the necessary process of smoothing, can grow exponentially and affect the distribution significantly at some later time. Also, it is not yet known how far unpredictable astronomical events, such as bursts of cosmic radiation, sunspots or meteoric showers, may affect the macro processes of the atmosphere. If they are capable of causing significant changes, it follows that weather is really indeterminate, for some at least of these phenomena are the result of occurrences which took place æons ago deep in the remote universe.

In routine forecasting, the weather prognosis is built around the time-extrapolation of the existing pressure fields, a process which is highly subjective. In deciding how the air masses will rearrange themselves in the course of the next twenty-four hours, the forecaster may be guided by the properties of a model, such as the Bjerknes depression, by con. siderations of the topography of the pressure surfaces, 
or by a set of private empirical rules which constitute his 'experience'. Whatever method is adopted, it is evident that good judgment and long practice are essentials in this difficult art. The mathematical method should be regarded as a means of indicating the most probable line of development. It seems that we should be unwise to expect more than this, at least for some years to come.

Ideally, any system of mathematical prediction should be based on conditions over the whole globe; but in practice, for a $24-\mathrm{hr}$. forecast in Britain, it is sufficient to restrict the calculations to the movement of air masses over a rectangle covering part of the eastern Atlantic and part of western Europe. The distribution of air pressure at the surface and at some considerable height in the troposphere is known tolerably well within this rectangle at any one time. With a conventional 'lid', say, at the tropopause, and the assumption that conditions do not change (or change according to some prescribed law) at the boundaries of the rectangle, the problem amounts to tracing the movements of the air inside the box formed by the rectangle, the vertical walls and the 'lid' in a period of, say, twenty-four hours. In other words, we set up a model atmosphere which obeys certain simplified forms of the laws of Nature, but we are not restricted to a rigid model of an anticyclone or depression in the sense used earlier.

This particular model, like many others, is a compromise. For some time to come meteorologists must be prepared to accept models which are not strictly logical, in that there is an arbitrary selection of certain features to be retained, while others are rejected, not necessarily because they are small but because their retention would make the model unworkable. As in mathematical physics generally, such models are idealizations, useful but not easily justified on strict mathematical grounds. It is important to realize that in such investigations there is no attempt to produce a forecast of weather. Attention is limited to an examination of the pressure and motion fields over a relatively small part of one hemisphere, in the middle latitudes. Even if the method were infallible (which it is not) the services of the meteorologist, as distinct from the mathe. matician, would still be required to 'put in the weather', a phrase which covers what may well be the most difficult part of the whole process of forecasting. It will be the responsibility of the forecaster, drawing on his experience, to accept or reject the answer provided by the mathematicians, and he must always recognize that the subjective element has not been eliminated by the mathematics but removed a stage farther back, to the initial postulates of the model.

The numerical method has been tried by the Meteorological Office, so far, on a few occasions only. The results are encouraging. The charts produced by the machine for the surface and the mid-troposphere have reproduced the main kinematical features of the real situation; they are about as good as those which were drawn by experienced forecasters on the dates in question. Thus, while it is far too early to claim that the value of the method is established, there are good grounds for continuing the research.

\section{Conclusions}

The fact that a weather forecast is essentially a statement of chances is not fully appreciated by the non-meteorologist, and some of the distrust with which the forecasts are regarded can be attributed to this misunderstanding.

The task of the meteorologist does not finish with the composition of the forecast-there is still the very important problem of conveying the results of the analysis to the public in simple and straightforward terms; and this part of the problem is almost as important as the examination of the physical processes at work.

\section{NATURAL MAGNETIZATION OF IGNEOUS AND SEDIMENTARY ROCKS}

1 DISCUSSION of recent developments in Great A Britain in the study of the permanent magnetization of rocks was held during January 8-9 in the Geology Department of the University of Birmingham. The chair was taken at the three sessions in turn by Prof. F. W. Shotton (University of Birming. ham), Prof. O. T. Jones (University of Cambridge) and Dr. J. McG. Bruckshaw (Imperial College of Science and Technology, London).

The permanent magnetization of rocks is due to the ferromagnetism of their iron oxide minerals. Dr. G. D. Nicholls (Department of Geology, Manchester), in summarizing what is known of their mineralogy, pointed out that they are often loosely classified as 'magnetite', but it is important to know their exact composition, on which their magnetic properties are very dependent. They occur naturally as solid solutions of hæmatite $\left(\mathrm{Fe}_{2} \mathrm{O}_{3}\right.$, rhombohedral), maghæmite $\left(\mathrm{Fe}_{2} \mathrm{O}_{3}\right.$, cubic), magnetite $\left(\mathrm{Fe}_{3} \mathrm{O}_{4}\right.$, cubic) and ilmenite $\left(\mathrm{FeTiO}_{3}\right.$, rhombohedral), of which the first three are ferromagnetic. The known range of solid solution is most simply expressed by the ternary $\mathrm{FeO}-\mathrm{Fe}_{2} \mathrm{O}_{3}-\mathrm{TiO}_{2}$ diagram, shown in Fig. 1 .

The full lines represent known solid solutions; the dotted line is conjectural but probably represents qualitatively the boundary of a region of no solid solution at magmatic temperatures. There is evidence that the size of this region increases with decreasing temperatures, leading to exsolution on cooling and the existence of two phases in the resulting mineral grains. In slowly cooled basic rocks minerals of the magnetite-ulvöspinel series seem to be most common, whereas in those which have been rapidly chilled minerals occur the compositions of which are inter-

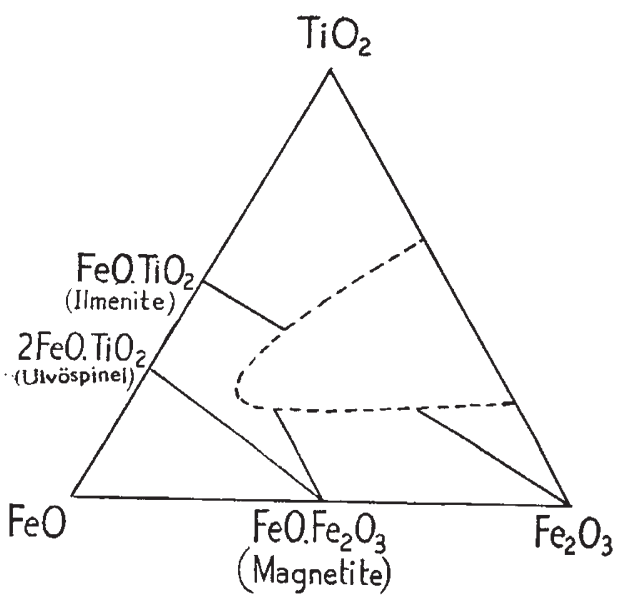

